\title{
sMEK1 promotes crosstalk between IRE1 and Akt signaling pathways: Evidence for a novel IRE1/sMEK1/Akt complex
}

\author{
Ozaira Qadri ${ }^{1}$, Samirul Bashir ${ }^{1}$, Mariam Banday ${ }^{1}$, Nazia Hilal ${ }^{1}$, and Khalid Majid Fazili ${ }^{1 \#}$ \\ ${ }^{\prime}$ Department of Biotechnology, University of Kashmir, Srinagar, India- 190006. \\ \#Corresponding author: Prof. Khalid Majid Fazili, PhD. \\ Department of Biotechnology \\ University of Kashmir, Srinagar, J\&K, India-190006. \\ Fax- +91 (194) 2272096 \\ (http://biotechnology.uok.edu.in/) \\ E-mail: fazili@kashmiruniversity.ac.in
}

\begin{abstract}
ER is facilitated with a dynamic cellular pathway namely Unfolded Protein Response (UPR): an adaptive signalling mechanism that maintains proteostasis in response to ER stress. IRE1 is one of the three transmembrane sensors of UPR with dual protein kinase and ribonuclease activities. IRE1 acts as a central molecule of UPR, which associates with a number of proteins that either regulate its activity or connect it to other pathways. Here, we report sMEK1 and Akt as novel interacting partners of IRE1 which associate to orchestrate the IRE1 and Akt signalling networks. Our study revealed that ER stress negatively regulates Akt through IRE1 protein. We found that IRE1/sMEK1/Akt form a ternary complex, which results in the dephosphorylation of Akt by protein phosphotase sMEK1 in presence of activated IRE1. Together, this study highlights the UPR/Akt link by delineating the molecular mechanism along with giving insights into the overall impact of this interaction.
\end{abstract}

Keywords: ER stress, UPR, IRE1, Akt, sMEK1

\section{Introduction}

Unfolded Protein Response (UPR) is a dynamic signalling network operating in endoplasmic reticulum [ER], which induces a transcriptional program coupled with intracellular signalling from the ER to the nucleus [1]. In higher eukaryotes, UPR is initiated by three main signalling proteins; Inositol requiring protein 1 (IRE1), double stranded RNA dependent Protein kinaselike ER kinase (PERK) and Activating transcription factor 6 (ATF6) [2]. Among these, IRE1 is the most evolutionary conserved and extensively studied arm of the UPR. IRE1 is a bifunctional enzyme with both the kinase and endoribonuclease activity, that either targets mRNA encoding the transcription factor X box-binding protein 1 (XBP1) or ER-targeted mRNAs in a process termed regulated IRE1-dependent mRNA decay (RIDD) [3]. The primary 
function of the UPR is to relieve cells from stress but leads to cell death in response to continued stress [4].

Several studies have put forth that UPR instead of being a unidirectional pathway communicates with a number of other pathways and sends signals multi-directionally [4]. One of the key players is IRE1 which acts as the protein docking platform and orchestrates with other pathways to relay the signal. This concept is known as 'UPRosome', where IRE1 is thought to act centrally and interact with multitude of proteins that can either activate/inactivate or connect it with other signalling networks [5-7].

In this context, one of the proteins revealed by our mass spectroscopy studies is SMEK1 (suppressor of MEK1) which is found to interact with activated IRE1 and interestingly has a role in modulating the $\mathrm{Akt} / \mathrm{mTOR}$ pathway. SMEK1 is a tumour suppressor protein also known as protein phosphatase 4 regulatory subunit 3 (PP4R3), a member of the PP2A subfamily, which has a conserved serine/threonine phosphatase domain [8-10]. sMEK1 acts as a novel pro-apoptotic protein while decreasing the expression of the AKT/mTOR pathway [11 -13]. These studies provide convincing evidence that sMEK1 is able to attenuate Akt/mTOR pathway. Also, there are evidences which point towards the communicating link between UPR and Akt pathways but they lack mechanistic details [14-16]. To this end, we investigated the functional link between IRE1 and Akt pathways. We found that IRE1 negatively regulates Akt phosphorylation through sMEK1. Our results revealed that both Akt and sMEK1 assemble in a complex with IRE1 which makes the basis for Akt regulation. Therefore, our results not only validate the connection between IRE1 and Akt pathways but also give a throughput understanding of the mechanism involved in the process.

\section{Material and Methods}

\subsection{Chemicals and Reagents}

HEK (Human Embryonic Kidney)-293T cell line, were obtained from the National Centre for Cell Sciences, Pune, India, Dulbecco's modified Eagles medium (Gibco by Life Technologies), Fetal bovine serum (Gibco by Life Technologies), penicillin-streptomycin solution (Gibco by Life Technologies), Tunicamycin (Tm) (Cal Biochem, USA), and Trypsin-EDTA (Gibco by Life Technologies). Antibodies used for Immunoblotting; anti-GST Antibody (Cell Signaling Technologies), IRE1 $\alpha$ (14C10) Rabbit mAb (Cell Signaling Technologies), anti-Akt antibody (Cell Signaling Technologies), anti-IRE1 (phospho S724) antibody (Cell Signaling Technologies), anti-HA (Cell Signaling Technologies), anti-sMEK1 (Santa Cruz 
Biotechnology, Santa Cruz, CA, USA) anti-Rabbit IgG Alkaline Phosphatase antibody (Sigma Aldrich), anti-mouse IgG Alkaline Phosphatase antibody (Sigma Aldrich), IRDye Goat antiRabbit IgG (LI-CORR Biosciences), IRDye Goat anti-Mouse IgG (LI-CORR Biosciences) were purchased from the respective cited companies. All other chemicals and reagents including cell culture media, chemicals like Tris-Base, SDS, 2-ME, and other solvents were obtained from Sigma-Aldrich, Invitrogen and HiMedia.

\subsection{Cell Culture and Treatments}

HEK293-T maintained in Dulbecco's Modified Eagle's Medium (DMEM) supplemented with penicillin and streptomycin (each 1\% v/v), 10\% FBS, grown in the humidified environment of $37^{\circ} \mathrm{C}$ temperature and $5 \% \mathrm{CO}$. Cells were treated with $6 \mu \mathrm{M}$ tunicamycin for UPR induction.

\subsection{Gene Cloning}

For recombinant expression in HEK293T cells, full- length human IRE1 and sMEK1 cDNAs were amplified from HEK293T cDNA by PCR and cloned into pcDNA3.1 and pEBGGST/pcDNA3.1 respectively. Akt-HA-pcDNA 3.1 was precured from Addgene. For yeast two hybrid analysis, IRE1 and sMEK1 genes were cloned in pGAD24 and pGBT9 yeast vectors respectively.

\subsection{Co-Immunoprecipitation}

For co-immunoprecipitation, cells were rinsed in phosphate-buffered saline (PBS) and dissolved in cell lysis NETN buffer [50mM Tris-Cl, 150mM NaCl, 1\% Glycerol, 1\% Np-40, $0.1 \%$ SDS, $5 \mathrm{mM}$ EDTA, $0.5 \%$ Sodium deoxycholate, $10 \mathrm{mM}$ NaF, $17.5 \mathrm{mM} \beta-$ glycerophosphate, 1X PIC)]. Lysates were then incubated with anti-IRE1 antibody or anti-GST antibody and precipitated using protein A-agarose (Invitrogen, Carlsbad, CA, USA). Precipitated proteins were separated by $10 \%$ sodium dodecyl sulfate polyacrylamide gel electrophoresis (SDS-PAGE), transferred to PVDF membrane and subjected to immunoblot analysis using either anti-HA or anti-GST antibodies.

\subsection{Yeast Two Hybrid Assay}

The IRE1 and sMEK1 genes were cloned into the pGAD24 and pGBT9 yeast vectors, respectively, then co-transformed into the AH109 yeast strain, which contains a His-reporter gene. GAL4 activation domain is found in pGAD24, while GAL4 transcription binding domain is found in pGBT9. SDL-T-Agar was patched with single colonies and cultured at $30^{\circ} \mathrm{C}$ for 
three days. When the transformant cultures grew, they were spotted on SDL-T-H'- media (SD complete media with leucine, tryptophan and histidine drop out). To analyze the growth on the plates, they were continually examined for 5-6 days. Likewise, pGAD24-Ire1 + pGBT9 vector and pGBT-sMEK1 + pGAD42 vector were co-transformed to create a negative control for the experiment. Since the promoter of the histidine biosynthesis gene responds to the transcription factor GAL4. As a result, the expression of His-gene will be dependent on the direct induction of GAL4 transcription factor via direct interaction between Ire1 and sMEK1, allowing colonies to thrive on $\mathrm{SDL}^{-} \mathrm{T}^{-} \mathrm{H}^{-} \mathrm{drop}$ out medium.

\subsection{Gene Silencing by small interfering RNA (siRNA knockdown)}

Oligonucleotides

containing

the

sequence

5'-

TGCATGTTCTCACTGCTCCTTTACTAGCAAATACAACAGAAGACAAACCTAGTAA AGATGATTTTCAGACTGCCCAACTATTGGCACTTGTATTGGAATTGTTAACATTTT GTGTGGAGCACCATACCTACCACATAAAGAACTACATTATTAATAAGGATATCCT CCGGAGAGTGCTAGTTCTTATGGCCTCGAAGCATGCTTTCTTGGCATTATGTGCC

CTTCGTTTTAAAAGAAAGATTATTGGATTAAAAGATGAGTTTTACAACCGCTACA TAATGAAAAGTTTTTTGTTTGAACCAGTAGTGAAAGCATTTCTCAACAATGGATC CCGCTACAATCTGATGAACTCTGCCATAATAGAGATGTTTGAATTTATTAGAGTG GAAGATATAAAATCATTAACTGCTCATGTAATTGAAAATTACTGGAAAGCACTG GAAGATG-3' were used (Mission esiRNA, Sigma) and transfected into cells using Lipofectamine 2000 reagent (Invitrogen) according to the manufacturer's instructions to silence endogenous sMEK1 expression. Mission siRNA fluorescent universal negative control \#1, Cyanine 3 was also transfected, acting as a negative control for siRNA knock down. Cells were seeded for $24 \mathrm{~h}$ and $48 \mathrm{~h}$ followed by transfection with $14000 \mathrm{ng}$ si-sMEK1. At $24 \mathrm{~h}$ after transfection, cells were prepared for immunoblot analysis.

\subsection{Western Blotting}

Cells were harvested and washed thrice with PBS $(\mathrm{pH}=7.4)$ and whole-cell lysates were prepared using the NP40 cell lysis buffer [50mM Tris-Cl, 150mM NaCl, 1\% Glycerol, 1\% Np40, 0.1\% SDS, 5mM EDTA, 0.5\% Sodium deoxycholate] which was supplemented by NaF (10mM), $\beta$ - Glycerophosphate (17.5mM), and Protease Inhibitor Cocktail (PIC) (1X). Protein concentrations were determined using the Bradford Assay [17]. An equal amount of protein $(20-30 \mu \mathrm{g})$ was electrophoresed by $12 \%$ SDS PAGE and transferred to a PVDF membrane. 
Membrane was blocked with 5\% BSA in 1XTBS buffer and incubated with primary antibody overnight at $4{ }^{\circ} \mathrm{C}$. The blot was then probed against the Mouse or Rabbit Secondary Antibody (LI-COR Odyssey, USA) with 1:5000 dilutions. The blot was analysed using LI-COR imaging system (LI-COR Biosciences, USA). Secondary antibody ALP conjugate (1:40,000 dilution) was also used and its blots were analysed on Gel doc system (BioRad, CA, USA).

\section{Results}

\section{1 sMEK1 directly interacts with IRE1}

A.

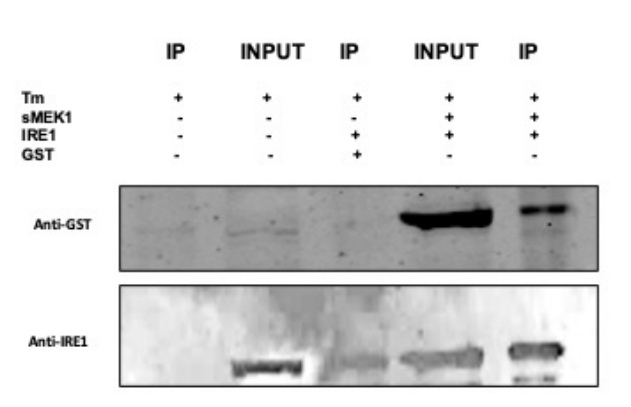

B.

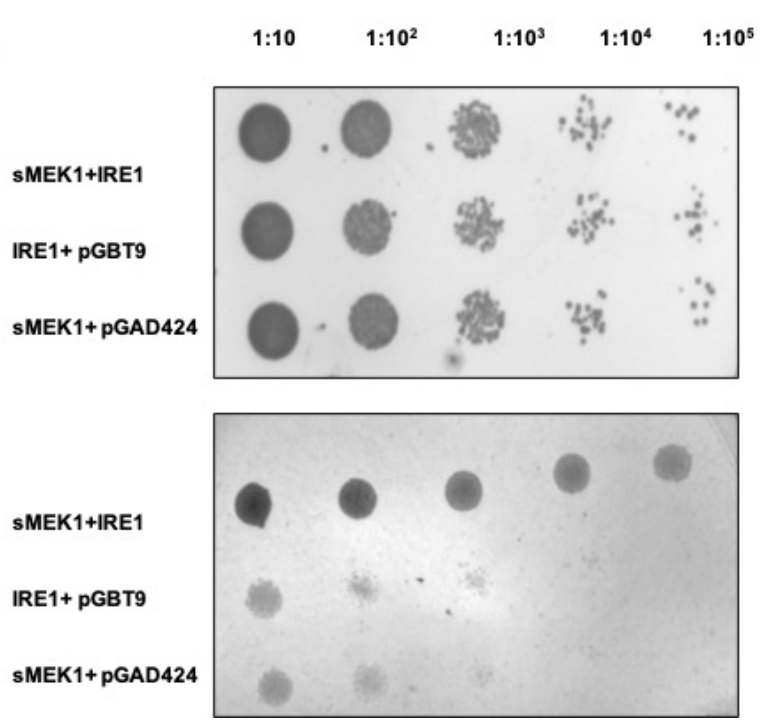

Figure1: Interaction of SMEK1 with IRE1; A) Co-immunoprecipitation of IRE1 with sMEK1. Immunoprecipitation (IP) was performed using an anti-IRE1 antibody and lysates from transfected HEK293T cells. After immunoprecipitation, precipitated proteins were immunoblotted using anti-GST antibody. lane 1,2 IP and Input for Cells only with no transfection; lane3 IP for GST-pEBG (vector only); lane 4,5 GST-pEBG-sMEKIand pcDNA3.1 IRE1 transfectants. B) Yeast Two Hybrid Assay showing direct interaction of IRE1 with sMEK1. pGAD24-Ire1 + pGBT9-sMEK1 represents colonies obtained from co-transfection of $p G A D-I R E 1$ and $p G B T 9$ plasmids. pGAD24 + pGBT9-sMEK1shows colonies obtained from co-transfection of pGAD24 and pGBT9sMEK1plasmids. pGAD-IRE1 + pGBT9 represent colonies obtained from co-transfection of $p G A D-I R E 1$ and pGBT9 plasmids. Dilution spotting on SDL-T $H^{-}$drop out media to select for positive interactors (Upper panel). Lower panel shows dilution spotting on YPD rich media. Dilutions were made up to $10^{-5}$.

Initially we identified sMEK1 as a potential IRE1 partner in the mass spectroscopic analysis (Data unpublished). To further explore the functional relevance involved in the IRE1/sMEK1 link, we first validated the interaction between the two proteins. We used a yeast two-hybrid system and co-immunoprecipitation assay to check for the interaction. Gene constructs of IRE1 (pcDNA3.1) and sMEK1 (GST-pEBG), vector only (GST-pEBG) were co-transfected into HEK293T cells. Then, immunopulldown was carried out by using anti-IRE1 antibody with lysates from both transfected cells. After immunoprecipitation, the precipitated proteins were immunoblotted using anti-GST [Fig. 1A]. To further confirm, the direct interaction between IRE1 and sMEK1 we carried out yeast two-hybrid assay. The IRE1 fused to the Gal4 
transcription activation domain and the sMEK1 to the binding domain were introduced into yeast cells. After obtaining independent transformant cells, cultures were spotted on the selection media (His-, Trp-, Leu-). Finally, the transformant cells containing the genes for both SMEK1 and IRE1 grew on selection media, thus proving the direct interaction between the two proteins. [Fig. 1B].

\subsection{IRE1 is crucial for sMEK1 mediated dephosphorylation of Akt}

A.

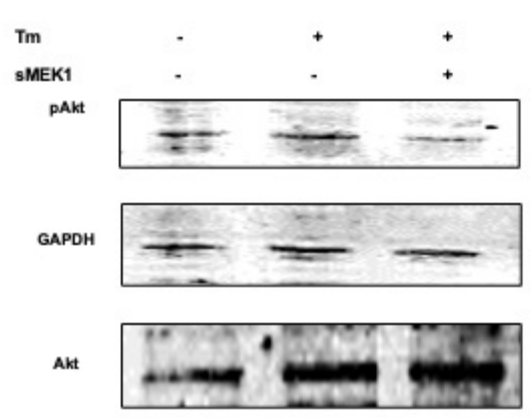

C.

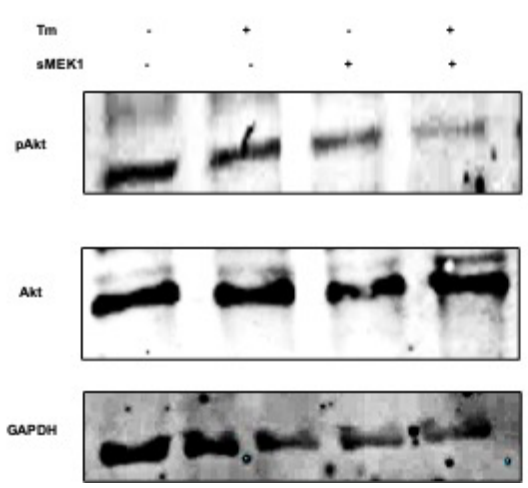

B.

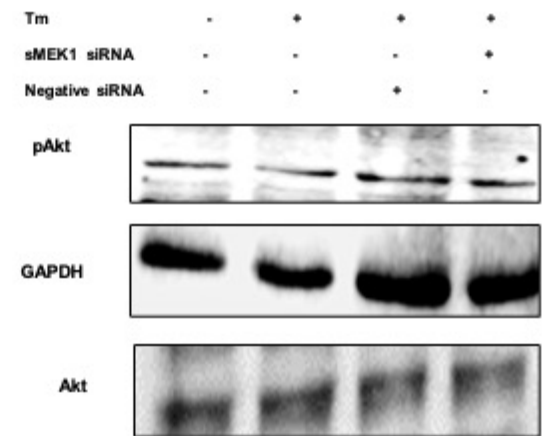

D.

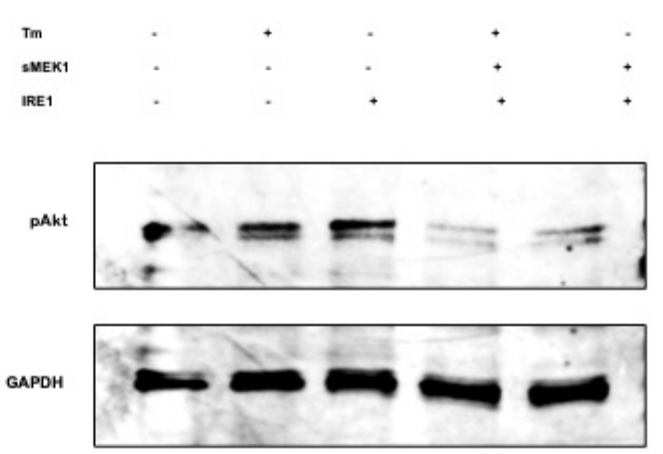

Figure 2 sMEK1 dephosphorylated Akt through IRE1 A) Analysis of Akt phosphorylation in smek1 overexpressing conditions. HEK293-T cells were transiently transfected for sMEK1-pcDNA3.1. After 36hr cells were stimulated with $6 \mu M$ tunicamycin for $4 \mathrm{hr}$. Levels of pAkt were checked by probing with Anti-pAkt antibody. B) Effect of pAkt under sMEK1 knockdown conditions. Cells were transfected with small interfering RNAs for $24 \mathrm{~h}$ for effective knockdown of SMEK1, and its effect on pAkt was analysed using western blotting. C) Analysis of Akt phosphorylation under different combinations of Tm $(6 \mu M, 4 h)$ and $s M E K 1$ overexpression. Akt phosphorylation levels were checked using Anti-pAkt antibody. D) Analysis of Akt phosphorylation in IRE1 and sMEKI overexpressed conditions. Cells were transfected for sMEK1-pcDNA3.1 and IRE1-pcDNA3.1 and Tm (6 $\mu M, 4 h)$. Cell lysates obtained from different treatment conditions were used for Immunoblotting against pAkt-antibody. All the Cell lysates subjected to Western Blotting had GAPDH as an endogenous control.

Considering the previous studies that hinted towards the relation between Akt and UPR $[15,16]$, we wanted to explore whether sMEK1 regulates Akt activity through UPR. It was found that the treatment with Tunicamycin (Tm) an ER stress inducers resulted in the dephosphorylation of Akt in presence of sMEK1 [Fig 2A], indicating the presence of sMEK1 as prerequisite to deactivate Akt [Fig. 2C]. Moreover, it was found that sMEK1 was only able to modulate Akt phosphorylation when UPR was active as induced by the treatment with 
Tunicamycin, confirming the role of UPR in Akt regulation. [Fig. 2C]. To further detect whether the effect was specifically through IRE1 we overexpressed IRE1 in HEK293T cells, such that only IRE1 arm is activated. It was found that SMEK1 regulation of Akt was through IRE1, where IRE1 overexpressed state was able to effectively deactivate Akt phosphorylation [Fig. 2D]. The above observations suggest that the suppression of Akt activity is ER stressdependent regulated by sMEK1 through IRE1 arm of UPR.

\subsection{Akt forms a ternary complex with sMEK1 and IRE1}

A.

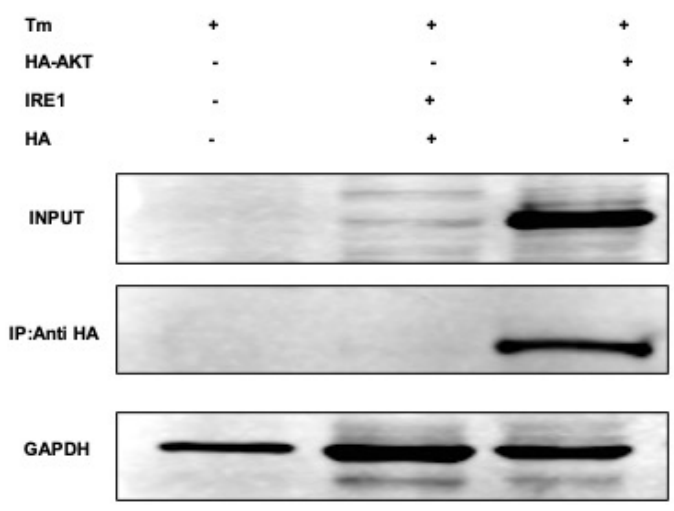

B.

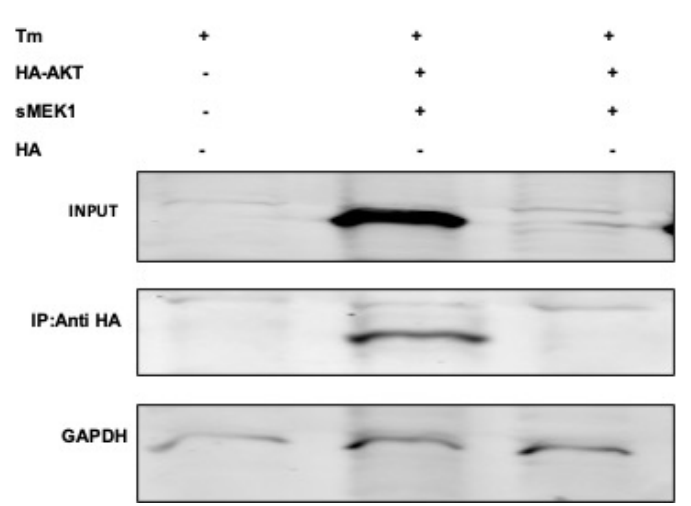

Figure3: Interaction of Akt sMEK1 and IRE1; A) Co-immunoprecipitation of IRE1 with Akt. Immunoprecipitation (IP) was performed using an anti-IRE1 antibody and lysates from transfected in HEK293T cells. After immunoprecipitation, precipitated proteins were immunoblotted using anti-HA antibody. lane 1, Cells only with no transfection; lane2, HA-pcDNA 3.1 (vector only) ; lane 3 Akt-HA-pcDNA3.1 and pcDNA3.1 IRE1 transfectants. B) Co-immunoprecipitation of sMEK1 with Akt. Immunoprecipitation (IP) was performed using an anti-sMEK1 antibody and lysates from transfected in HEK293T cells. After immunoprecipitation, precipitated proteins were immunoblotted using anti-HA antibody. lane 1, Cells only with no transfection lane 2 Akt-HApcDA3.1 and pcDNA3.1 sMEK1, lane3, HA-pcDNA 3.1 (vector only) transfectants. GAPDH was used as internal loading control.

In continuation with above observations that clearly indicate sMEK1 interaction with IRE1 resulted in downstream effect on Akt phosphorylation, we extended our study to check if the three protein IRE1, sMEK1 and Akt are in close association. For this, we carried out Coimmunoprecipitation assay. Gene constructs of IRE1 (pcDNA3.1) and Akt (HA-pcDNA), sMEK1 (pcDNA 3.1) and Akt (HA-pcDNA), were co-transfected into HEK293T cells. Coimmunoprecipitation was carried out by using anti-IRE1/anti-sMEK1 antibody with lysates from both transfected cells. After immunoprecipitation, the precipitated proteins were immunoblotted using anti-HA antibody [Fig. 3A,3B]. Interestingly, we found that Akt interacts with both IRE1[Fig. 3A] and sMEK1 [Fig. 3B]. With these observations we concluded that IRE, sMEK1 and Akt associate with each other in a close proximity to regulate Akt activity. 


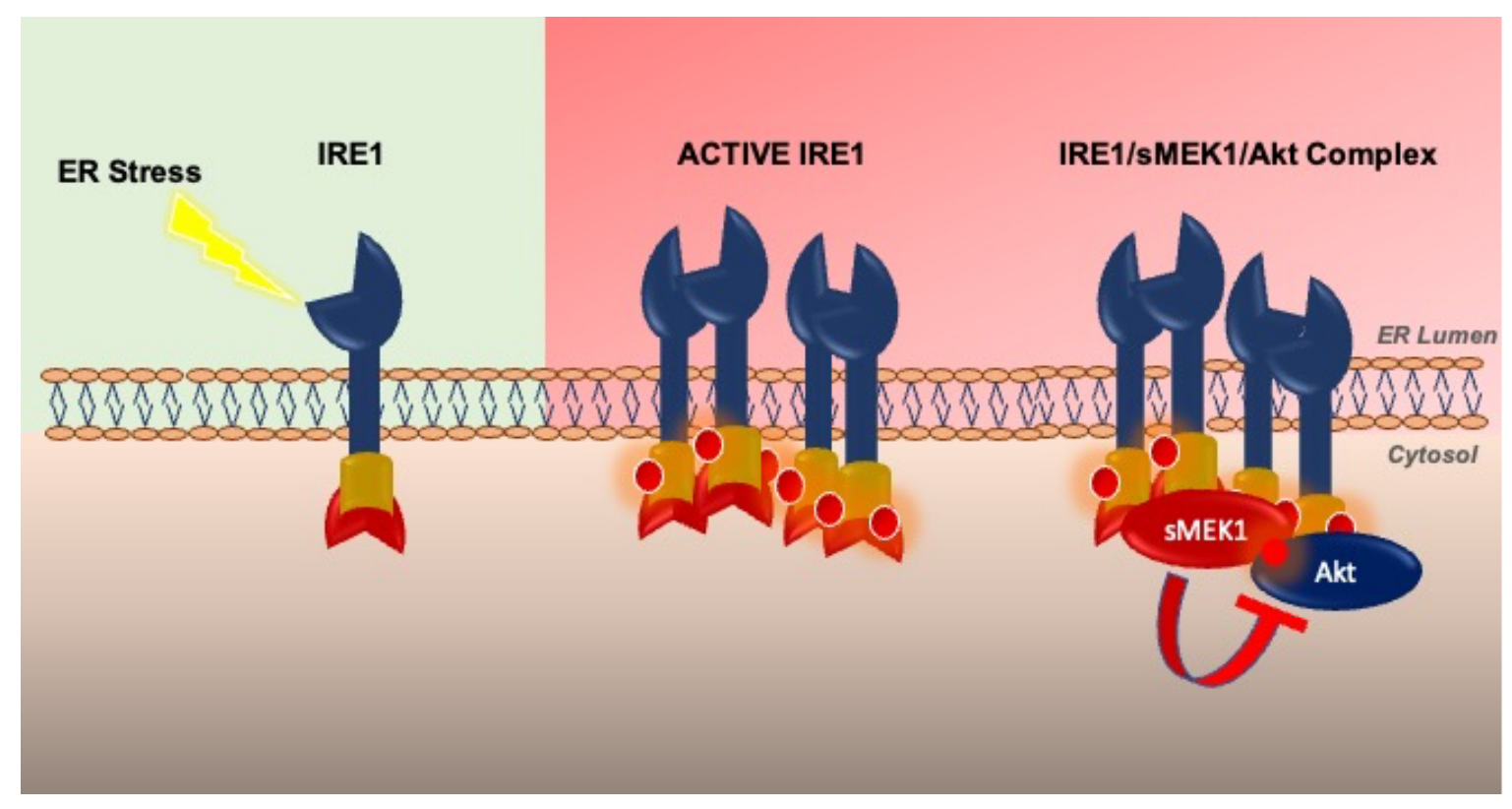

Figure4: Proposed model for relay of signal from IRE1 sensor of UPR to Akt-signalling via sMEK1. Under ER stress the monomeric IRE1 comes together to activate its trans-autophosphorylation and thereby its endoribonuclease activity. The active IRE1 is able to dock sMEK1 and Akt such that three proteins come in close proximity. This close association of IRE1/sMEK1/Akt leads to the deactivation of Akt by protein phosphatase sMEK1.

\section{Discussion}

IRE1 acts as a central pathway which orchestrates with different binding partners, leading to the definition of a dynamic signalling platform that has been referred to as the 'UPRosome', in which many regulatory and adaptor proteins assemble to activate and modulate downstream responses [7]. An array of proteins are found to physically interact with IRE1 either serving as its modulators or functional links with other pathways or simply the structural stabilizers of IRE1 [3]. Therefore, to further understand the role of IRE1 interactome, we found sMEK1 as one of the possible interacting partner of IRE1. To validate this interaction we carried out coimmunoprecipitation and yeast two hybrid assays and found that SMEK1 was able to directly interact with IRE1.

Besides, these physical interactors there exists a functional crosstalk between IRE1 and other cellular signalling pathways including one of important cellular signalling pathway, the mammalian target of rapamycin (mTOR). An increasing number of evidences are cited so far which establishes a candid link between the two. These studies propose that mTOR selectively suppresses IRE1 $\alpha$ activation by AKT-mTOR-mediated attenuation that controls the dynamics of IRE1 deactivation by regulating ER-mitochondria physical contacts and the 
phosphorylation state of IRE1 [15]. And reciprocally the other set of studies put forth that prolonged ER stress negatively regulates mTOR attributing to its inactivation and induction of apoptosis via autophagy [16]. The idea evolving from these studies is that there exists a functional crosstalk between UPR and Akt pathways which may act antagonistically but integrate and transduce to produce a substantial biological outcome and consequently act in determining the fate of a cell. However, this link lacks proper molecular outline where a little is known about the molecular mediators involved in this connection. In this study, we found that sMEK1 acts as the molecular bridge between two pathways and the trio IRE1/sMEK1/Akt share a functional link. Our results clearly indicated that ER stress leads to the docking of sMEK1 and Akt on IRE1 in a way that sMEK1 can dephosphorylate Akt [Fig. 4]. Therefore, this study reveals the connection between the two pathways in an interesting way that it unravels a number of aspects regarding the crosstalk, regulation and the relay of signal between them. 


\section{References}

1. Hetz C, Martinon, F, Rodriguez D, Glimcher, LH (2011). The unfolded protein response: integrating stress signals through the stress sensor IRE1 $\alpha$. Physiol. Rev. 91(14):1219-1243

2. Schroder M, Kaufman RJ (2005). The mammalian unfolded protein response. Annu Rev Biochem. 74:739-89.

3. Bashir S, Banday M, Qadri O, Bashir A, Hilal N, Nida-i-Fatima, Rader S, Fazili KM, (2021), The molecular mechanism and functional diversity of UPR signaling sensor IRE1, Life Sciences, , Volume 265,, 2021, 118740

4. Jäger R, Bertrand MJ, Gorman AM, Vandenabeele P, Samali A (2012). The unfolded protein response at the crossroads of cellular life and death during endoplasmic reticulum stress. Biol Cell. 104(5):259-70.

5. Hetz C, Martinon, F, Rodriguez D, Glimcher, LH (2011). The unfolded protein response: integrating stress signals through the stress sensor IRE1 $\alpha$. Physiol. Rev. 91(14):1219-1243

6. Urra H, Pihán P, Hetz C (2020). The UPRosome - decoding novel biological outputs of IRE1 $\alpha$ function. J.Cell Sci. 11;133(15)

7. Hetz C, Glimcher, LH (2009). Fine-tuning of the unfolded protein response: Assembling the IRE1 $\alpha$ interactome. Mol. Cell 35(5):551-561.

8. Chen GI, Tisayakorn S, Jorgensen C, D'Ambrosio LM, Goudreault M, Gingras AC (2008) PP4R4/KIAA1622 forms a novel stable cytosolic complex with phosphoprotein phosphatase 4, J. Biol. Chem. 283: 29273-29284.

9. Chowdhury D, Xu X, Zhong X, Ahmed F, Zhong J, Liao J, Dykxhoorn DM, Weinstock DM, Pfeifer GP, Lieberman J. (2008) A PP4-phosphatase complex dephosphorylates gamma- H2AX generated during DNA replication, Molecular Cell, 31: 33-46.

10. Nakada S, Chen GI, Gingras AC, Durocher D., (2008) PP4 is a gamma H2AX phosphatase required for recovery from the DNA damage checkpoint, EMBO Reports,;9: 1019-1026,

11. Byun HJ, Kim BR, Yoo R, Park SY, Rho SB. (2012) sMEK1 enhances gemcitabine anticancer activity through inhibition of phosphorylation of Akt/mTOR, Apoptosis , 17: 10951103 ,

12. Kim BR, Yoon K, Byun HJ, Seo SH, Lee SH and Rho SB, (2014) The anti-tumor activator sMEK1 and paclitaxel additively decrease expression of HIF-1 $\alpha$ and VEGF via mTORC1S6K/4E-BP-dependent signaling pathways, Oncotarget, 15;5(15):6540-51, 
13. Kim BR, Seo SH, Park MS, Lee SH, Kwon Y and Rho SB, (2015) sMEK1 inhibits endothelial cell proliferation by attenuating VEGFR-2-dependent-Akt/eNOS/HIF-1 $\alpha$ signaling pathways. Oncotarget. 13;6(31):31830-43.

14. Kato H, Nakajima S, Saito Y, Takahashi S, Katoh R \& Kitamura M (2011) mTORC1 serves ER stress-triggered apoptosis via selective activation of the IRE1-JNK pathway, Cell Death and Differentiation. 19(2):310-20

15. Sanchez-Alvarez M, Del Pozo MA, Bakal C (2017) AKT-mTOR signaling modulates the dynamics of IRE1 RNAse activiity by regulating ER-mitochondria contacts, Scientific Reports. 8(1):6476.

16. Qin L, Wang Z, Tao L and Wang Y, ER stress negatively regulates (2010) AKT/TSC/mTOR pathway to enhance autophagy, Autophagy 6:2, 239-247.

17. Bradford MM. (1976) A rapid and sensitive method for the quantitation of microgram quantities of protein utilizing the principle of protein-dye binding. Anal Biochem. 72:24854. 